\title{
Effects of carbon nanofiber on physiology of Drosophila
}

\author{
This article was published in the following Dove Press journal: \\ International Journal of Nanomedicine \\ 21 May 2015 \\ Number of times this article has been viewed
}

\section{Shin-Hae Lee ${ }^{1, *}$ \\ Hye-Yeon Lee ${ }^{1, *}$ \\ Eun-Ji Lee',* \\ Dongwoo Khang ${ }^{2}$ \\ Kyung-Jin Min'}

'Department of Biological Sciences, Inha University, Incheon, Republic of Korea; ${ }^{2}$ Department of Molecular Medicine, Graduate School of Medicine, Gachon University, Incheon, Republic of Korea

*These authors contributed equally to this work
Correspondence: Kyung-Jin Min Department of Biological Sciences, 100 Inha Street, Inha University, Incheon 402-75I, Republic of Korea

Tel +82328608193

Fax +82 328607690

Email minkj@inha.ac.kr
Abstract: As nanomaterials are now widely utilized in a wide range of fields for both medical and industrial applications, concerns over their potential toxicity to human health and the environment have increased. To evaluate the toxicity of long-term exposure to carbon nanofibers (CNFs) in an in vivo system, we selected Drosophila melanogaster as a model organism. Oral administration of CNFs at a concentration of $1,000 \mu \mathrm{g} / \mathrm{mL}$ had adverse effects on fly physiology. Long-term administration of a high dose of CNFs $(1,000 \mu \mathrm{g} / \mathrm{mL})$ reduced larval viability based on the pupa:egg ratio, adult fly lifespan, reproductive activity, climbing activity, and survival rate in response to starvation stress. However, CNFs at a low concentration $(100 \mu \mathrm{g} / \mathrm{mL}) \mathrm{did}$ not show any significant deleterious effect on developmental rate or fecundity. Furthermore, long-term administration of a low dose of CNFs $(100 \mu \mathrm{g} / \mathrm{mL})$ increased lifespan and climbing ability, coincident with mild reactive oxygen species generation and stimulation of the antioxidant system. Taken together, our data suggest that a high dose of CNFs has obvious physiological toxicity, whereas low-dose chronic exposure to CNFs can actually have beneficial effects via stimulation of the antioxidant defense system.

Keywords: toxicity, Drosophila melanogaster, lifespan, reactive oxygen species

\section{Introduction}

Nanomaterials are defined as materials with size dimensions on the nanoscale. Nanomaterials are widely utilized in a wide variety of fields for both medical and industrial applications, such as fillers, catalysts, semiconductors, cosmetics, microelectronics, tissue engineering, drug delivery, gene therapy, and biosensor technology. ${ }^{1-4}$ Accordingly, concerns over their potential toxicity to human health and the environment have increased. The small size of nanomaterials grants unique physicochemical properties such as high conductivity, strong optical scattering properties, strong absorbance, and ease of functionalization, but also induction of toxicological aspects. ${ }^{5}$

Among various nanomaterials, fibrous nanomaterials such as carbon nanotubes and carbon nanofibers (CNFs) have unique electronic and mechanical properties due to their tubular structure. Concerns over the toxicity of fibrous nanomaterials have been raised due to their structural similarity with asbestos, although their chemical composition and physic-mechanical properties are reported to be very different from asbestos. ${ }^{6}$ In addition, CNFs possess distinct properties from carbon nanotubes. ${ }^{7}$ Structurally, singlewalled carbon nanotubes are composed of a rolled-up cylindrical sheet of graphene, whereas CNFs are formed from stacked graphene nanocones. ${ }^{8}$ CNFs are strong and flexible filaments ranging from 70 to $200 \mathrm{~nm}$ in diameter and 10-100 $\mu \mathrm{m}$ in length, and they are usually used in advanced composite materials to improve strength, stiffness, durability, electrical conductivity, and heat resistance. ${ }^{9} \mathrm{CNF}$ are cost-effective compared to carbon nanotubes, and thus their commercial applicability has grown 
exponentially. Despite the widespread use of CNFs, toxicological studies on fibrous nanomaterials have mainly focused on carbon nanotubes. Furthermore, among the few studies that have evaluated CNF toxicity, only in vitro-cultured cell models have been used. ${ }^{7,10,11}$

Although nanomaterial-mediated toxicity has been largely attributed to the generation of free radicals, the exact molecular mechanism underlying reactive oxygen species (ROS) generation by nanoparticles remains unclear. ${ }^{12-14}$ Flies exposed to silver nanoparticles are known to show depleted levels of glutathione (GSH) as well as increased antioxidant enzyme SOD and CAT activities. ${ }^{15}$ In addition, coadministration of silver nanoparticles with an antioxidant such as vitamin $\mathrm{C}$ or vitamin $\mathrm{C}$ palmitate has been shown to significantly increase survivorship and development in flies. ${ }^{16}$ These reports suggest that the primary mechanism behind the toxicity of silver nanoparticles is induction of oxidative stress. Furthermore, titanium dioxide nanofibers are reported to induce oxidative stress-mediated cytotoxicity and apoptosis in HeLa cells, ${ }^{17}$ and CNFs are known to increase intracellular ROS production in Schwann cells. ${ }^{10}$

Although nanomaterial toxicity has been investigated, there have been only a few reports on the long-term effects of exposure to carbon nanomaterials in an in vivo model system. Drosophila melanogaster has attracted attention as a model system for evaluating the toxicities of artificial materials. ${ }^{18}$ Drosophila has many beneficial aspects, including ease of handling, various genetic tools, and a short lifespan. ${ }^{19}$ Recent studies have demonstrated nanomaterial toxicity in a D. melanogaster model system. Specifically, long-term exposure of $D$. melanogaster to silver nanoparticles during development has been shown to increase genetic instability related to somatic recombination as well as perturb copper homeostasis, resulting in impaired body pigmentation..$^{20,21}$ Short-term exposure of Drosophila larvae to silver nanoparticles is associated with induction of oxidative stress and apoptosis. ${ }^{15}$ In addition, long-term exposure to gold nanoparticles $(12 \mu \mathrm{g} / \mathrm{g})$ was reported to reduce lifespan and fertility in flies, ${ }^{22}$ depending on nanoparticle concentration rather than size. ${ }^{23}$ On the other hand, several reports have suggested that nanomaterials actually have no adverse effects in Drosophila. Functionalized single-walled carbon nanotubes with a hydroxyl group, organically modified silica nanoparticles, and gallium phosphide nanowires have all been reported as having no significant adverse effects on Drosophila development or adult lifespan. ${ }^{24-26}$ In addition, gellan gum-polyethylenimine nanocomposites show no significant effects on Drosophila survival, ${ }^{27}$ and long-term exposure to insulin-small lipid nanoparticles developed for insulin delivery is reported to be nontoxic. ${ }^{28}$

In the current study, we evaluated the physiological effects of long-term exposure to CNFs using D. melanogaster. CNFs at a concentration of $1,000 \mu \mathrm{g} / \mathrm{mL}$ showed adverse effects on development rate and lifespan, whereas $100 \mu \mathrm{g} / \mathrm{mL}$ of CNF supplementation showed hormetic beneficial effects on Drosophila physiology.

\section{Materials and methods Scanning electron microscopy}

CNFs (PR-24-XT-OX, low-crystalline, $100 \mathrm{~nm}$ diameter, $45 \mathrm{~m}^{2} / \mathrm{g}$ surface area) were obtained from Pyrograf Products, Inc. (Cedarville, OH, USA). CNF powder was attached on an aluminum mount with double-stick carbon tape and sputtercoated with platinum. Images were collected on a Hitachi S4300 field emission scanning electron microscope.

\section{Drosophila stocks and husbandry}

Canton-S wild-type flies were cultured and reared at $25^{\circ} \mathrm{C}$ and $65 \%$ humidity on a 12:12-hour light:dark cycle. Normal cornmeal-sugar-yeast (CSY) media (5.2\% cornmeal, $11 \%$ sugar, $2.6 \%$ instant yeast, $0.5 \%$ propionic acid, $0.2 \%$ methyl4-hydroxybenzoate [Sigma-Aldrich Co., St Louis, MO, USA], and $0.8 \%$ agar) were used to culture and rear the parent flies.

\section{Supplementation of CNFs}

Following previous reports on nanomaterial supplementation to flies, ${ }^{15,25,29-31} \mathrm{CNFs}$ were suspended in ethanol $(0.5,5 \%$ $\mathrm{wt}$ ), and stock CNF solutions were added to normal CSY or sucrose-yeast (SY) fly media (10\% sugar, 10\% instant yeast, $0.2 \%$ methyl-4-hydroxybenzoate, $0.5 \%$ propionic acid, and $0.8 \%$ agar) to make food with a final CNF concentration of 100 or $1,000 \mu \mathrm{g} / \mathrm{mL}(0.01$ or $0.1 \% \mathrm{wt})$. Parent flies reared on normal CSY media were transferred to CNF-containing CSY food in order to lay eggs for 24 hours. Newly eclosed F1-generation adults developed on CNF-containing CSY food were collected over 24 hours and used in all experiments after pre-feeding with a CNF-containing SY diet.

\section{Measurement of viability in developmental stages}

For larval viability, parent flies reared on normal CSY media were transferred to CNF-containing CSY food in order to lay eggs for 16 hours. After egg deposition, ten eggs were collected and transferred to fresh vials containing CSY diet with or without CNFs. The number of pupae was recorded at the time point when additional pupae no longer emerged. 
For pupal viability, the vials used for measurement of larval viability were maintained at $25^{\circ} \mathrm{C}$. The number of newly eclosed adult flies was recorded at the time point when all flies had hatched. Thirty replicates were established for each CNF dose.

\section{Crystal cell assay}

Parent flies reared on normal CSY media were transferred to CNF-containing CSY food in order to lay eggs for 24 hours. At the third instar stage (L3), larvae were collected and incubated at $70^{\circ} \mathrm{C}$ for 10 minutes to induce rupture of crystal cells, followed by release of enzymes leading to melanin production. Melanized dots were counted in abdominal segments $\mathrm{A} 6, \mathrm{~A} 7$, and $\mathrm{A} 8$.

\section{Lifespan assays}

Newly eclosed Canton-S adult flies developed on CNFcontaining CSY food or standard CSY food were collected over 48 hours and randomly assigned to $500 \mathrm{~cm}^{3}$ demography cages at a final density of 100 female and 100 male flies per cage. The vials containing fresh SY food with or without CNF were affixed to separate cages and changed every 2 days, after which dead flies were removed and recorded. Three replicate cages were established for each CNF dose. Experiments were conducted twice. Age-specific mortality rate $\left(\mu_{t}\right)$ was estimated by the Gompertz equation:

$$
\mu_{t}=-\ln \left(P_{t}\right)
$$

where $P_{t}$ is the probability of an individual alive at age $t-1$ surviving to age $t .{ }^{32}$

\section{Measurement of feeding rate}

Newly eclosed adult flies developed on CNF-containing CSY food were pretreated with CNFs for 7 days. After starvation for 6 hours, ten single-sex flies were transferred into a new vial containing the same diet and bromophenol blue dye $(0.05 \% \mathrm{wt} / \mathrm{vol})$ for 12 hours. The anesthetized flies were washed with phosphate-buffered saline and homogenized in $0.5 \mathrm{~mL}$ of distilled water. The absorbance of homogenate was measured at $595 \mathrm{~nm}$ using a spectrophotometer. Ten replicate vials were tested per treatment.

\section{Measurement of fecundity}

Within the first 24 hours of eclosion, adult flies developed on CNF-containing CSY food were collected, and each vial was set up at a density of two males and one female. Flies were transferred into new vials containing fresh SY food with or without CNF daily, and the number of eggs laid by each female was evaluated for 10 days. Fifteen replicate vials were tested per treatment.

\section{Measurement of physical activity}

To measure physical activity, adult flies developed on CNFcontaining CSY food were fed SY diets containing the same concentration of CNFs for 1 or 3 week(s) prior to performing a vertical climbing assay. Flies were collected under brief $\mathrm{CO}_{2}$ anesthesia (1-2 minutes) and allowed to recover for at least 18 hours. Ten single-sex flies were loaded into the apparatus for vertical climbing assay and tapped on a tabletop three times in rapid succession to initiate negative geotaxis responses. Positions of flies in the tubes were captured by digital images taken 4 seconds after initiating the behavior. The flies were assessed in consecutive trials separated by 1 minute of rest. Five replicates for each treatment and four trials were used in all experiments.

\section{Stress resistance test}

For starvation stress, flies developed on CNF-containing CSY food were pretreated with the same concentration of CNFs for 7 days, after which flies were kept in vials (ten single-sex flies per vial) containing $0.8 \%$ agar and transferred into fresh vials containing agar every 6 hours. Dead flies were scored after each transfer. Fifteen replicates were established.

\section{Measurement of intracellular GSH/GSSG level}

Total GSH and GSH disulfide (GSSG) levels were measured in a recycling assay using a commercially available GSH/ GSSG kit (GT40; Oxford Biomedical, Oxford, MI, USA). Briefly, 20 male flies were homogenized in ice-cold 5\% metaphosphoric acid solution, and supernatants were used for measurement of total GSH and GSSG levels based on extent of 2-nitro-5-thiobenzoic acid formation monitored at $415 \mathrm{~nm}$ for 10 minutes. The amounts of GSH and GSSG were calculated from a GSSG standard curve $(\mu \mathrm{M})$, as detailed in the manufacturer's instructions.

\section{Real-time quantitative polymerase chain reaction}

For real-time quantitative polymerase chain reaction (qPCR) in larvae, eggs were developed on CNF-containing food, and total RNAs from 13 wandering third-instar larvae were extracted using RNAiso (Takara Bio Inc., Otsu, Japan). For real-time qPCR in adult flies, total RNAs were extracted using RNAiso from 15 male flies after CNF feeding for 7 days. 
Total RNAs $(2 \mu \mathrm{g})$ were reverse-transcribed using MMLV reverse transcriptase (Promega Corporation, Fitchburg, WI, USA). qPCR was performed using the Prism7500 Sequence Detection System (Thermo Fisher Scientific, Waltham, MA, USA) and SYBR Green (Thermo Fisher Scientific) following the manufacturer's instructions. All experiments were repeated at least three times, and the data are presented as the mean \pm the standard error of the mean. The oligonucleotide sequences for defensin, ${ }^{33}$ superoxide dismutase (SOD) $1,{ }^{34}$ $S O D 2,{ }^{35}$ and catalase $(\mathrm{Cat})^{34}$ were previously reported. Ribosomal protein $49(\mathrm{Rp} 49)^{36}$ was used as an internal control.

\section{Statistical analysis}

Statistical log-rank tests for the demographic data were carried out using survival models in the JMP statistical package (SAS Institute Inc., Cary, NC, USA). For the analysis of variance and $t$-test, we used Excel in Microsoft Office 2010.

\section{Results}

\section{Effects of CNFs on Drosophila viability in developmental stage}

To characterize CNFs used in this study, we measured the diameter and length of CNFs using field emission scanning electron microscopy. CNF diameters ranged from 150 to $200 \mathrm{~nm}$, and length of CNFs was approximately 2-15 $\mu \mathrm{m}$ (Figure 1). To investigate CNF toxicity, we first measured the viability of larvae supplemented with CNF suspension at a concentration of 100 or $1,000 \mu \mathrm{g} / \mathrm{mL}$. Larval viability, defined as the number of pupae formed per egg, significantly decreased upon feeding with $1,000 \mu \mathrm{g} / \mathrm{mL}$ of $\mathrm{CNF} \operatorname{diet}(5.58 \%$ decrease, $P<0.05$ ), whereas viabilities of $100 \mu \mathrm{g} / \mathrm{mL}$ of $\mathrm{CNF}$ diet cohorts were unchanged (Figure 2A). Pupal viability, defined as the number of adults eclosed per pupae formed, was unchanged at both CNF concentrations (Figure 2B).

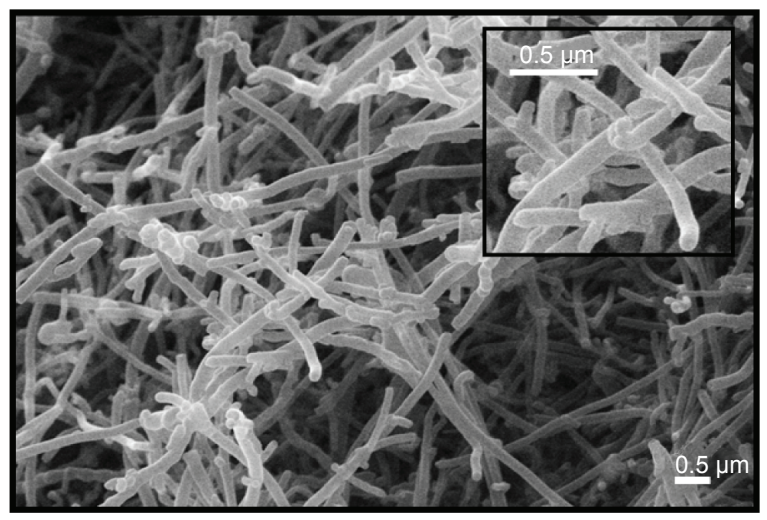

Figure I Scanning electron microscopic images of carbon nanofiber (CNF). Notes: The diameter of CNFs is approximately 150-200 nm, and the length of CNFs is approximately $3-15 \mu \mathrm{m}$. The scale bars correspond to $0.5 \mu \mathrm{m}$.
In addition, developmental time was slightly delayed with $1,000 \mu \mathrm{g} / \mathrm{mL}$ of CNF diet (data not shown). The external appearance of flies reared on CNF food was not significantly altered compared to that of control, except for dark masses considered to be CNF aggregation in the body cavities of larvae and adults (Figure 2C [arrows]). Furthermore, black spots appeared in segments of larvae reared on $1,000 \mu \mathrm{g} / \mathrm{mL}$ of CNF diet, suggesting that ingested CNFs were absorbed by flies and induced a biological response (Figure 2C [arrow heads]). We next analyzed the immune response of larvae exposed to CNFs, as black spots shown in larval segments were considered as immune granules. For this, we observed the number of crystal cells, which are a type of blood cell produced upon immune challenge in flies that can be visualized upon heat exposure of larvae. ${ }^{25}$ The number of crystal cells significantly increased upon ingestion of $100 \mu \mathrm{g} / \mathrm{mL}$ of CNFs (Figure 2D). Furthermore, expression of defensin, an antimicrobial peptide related to innate immunity of flies, increased upon ingestion of $100 \mu \mathrm{g} / \mathrm{mL}$ of CNFs (Figure 2E). This result indicates that CNFs affected the viability of Drosophila throughout its developmental stages and stimulated the immune response.

\section{Effects of CNFs on Drosophila lifespan}

We next investigated the effects of long-term exposure to CNFs on the adult fly lifespan. Newly eclosed flies were fed the same concentrations of CNF diet during development, and the number of survivors was counted every 2 days until all flies died. Survival analyses were performed based on the number of deaths recorded and evaluated by the Mantel-Cox log-rank test. The lifespan of flies supplemented with $1,000 \mu \mathrm{g} / \mathrm{mL}$ of CNFs significantly decreased in both sexes (Figure 3A). The mean lifespan of control male flies was $33.6 \pm 0.78$ days, whereas that of $1,000 \mu \mathrm{g} / \mathrm{mL}$ of CNF-supplemented male flies was 25.04 \pm 0.89 days (25.47\% decrease compared to control, log-rank test, $\left.\chi^{2}=37.04, P<0.0001\right)$. For female flies, the mean lifespan of control was $29.36 \pm 0.77$ days, whereas that of $1,000 \mu \mathrm{g} / \mathrm{mL}$ of CNF-supplemented flies was $21.86 \pm 0.74$ days $(25.56 \%$ decrease compared to control, log-rank test, $\chi^{2}=35.39$, $P<0.0001)$. Consistent with the survival data, the age-specific mortality rate of flies supplemented with $1,000 \mu \mathrm{g} / \mathrm{mL}$ of CNFs significantly increased in both sexes (Figure 3B). However, the lifespan of $100 \mu \mathrm{g} / \mathrm{mL}$ of CNF-supplemented flies increased in both sexes. The mean lifespan of $100 \mu \mathrm{g} / \mathrm{mL}$ of CNF-supplemented male flies was $38.01 \pm 0.79$ days (13.11\% increase compared to control, log-rank test, $\chi^{2}=14.76, P<0.0001$ ), whereas the mean lifespan 


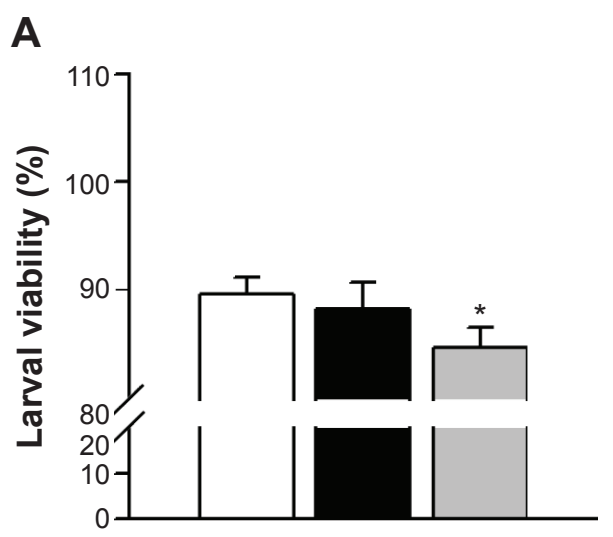

C

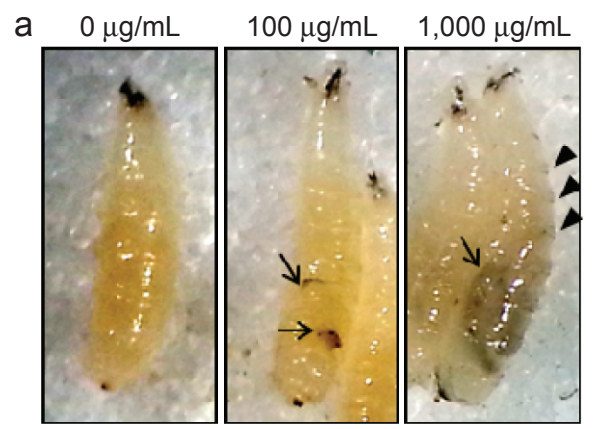

D

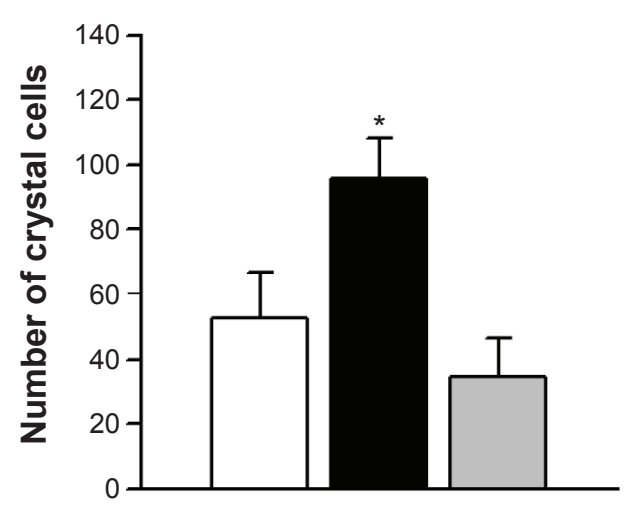

B

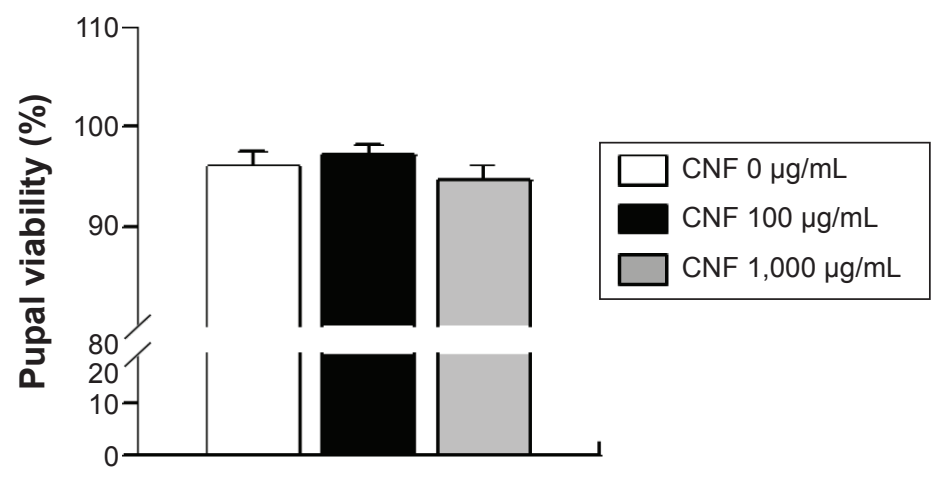

CNF

b

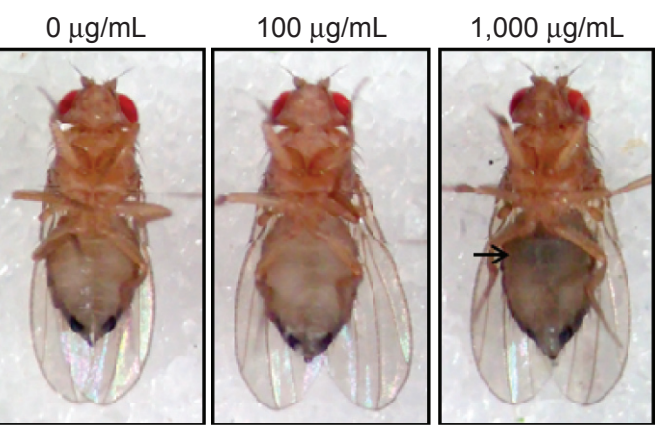

E

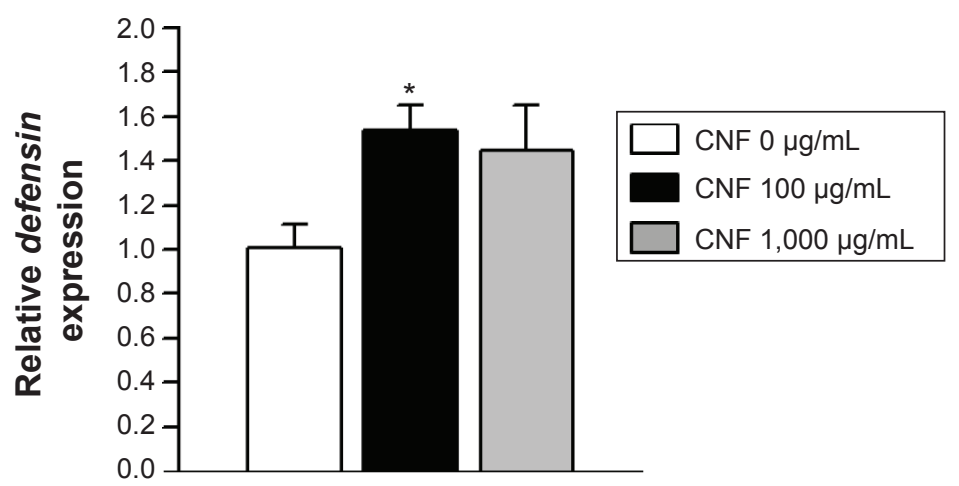

Figure 2 Effects of CNFs on larval and pupal viability in Drosophila.

Notes: (A) Larval viability was determined based on the pupa:egg ratio. Oral administration of CNFs at a dose of $100 \mu \mathrm{g} / \mathrm{mL}$ did not significantly affect larval viability (black bar), whereas $1,000 \mu \mathrm{g} / \mathrm{mL}$ of $\mathrm{CNF}$ administration significantly reduced larval viability (gray bar) compared to the control (white bar). $* P<0.05$. (B) Pupal viability was determined based on the eclosed adult:pupa ratio. CNF administration at both 100 and I,000 $\mu \mathrm{g} / \mathrm{mL}$ did not significantly alter pupal viability. (C) Microscopic images of larvae (a) and adult flies (b) reared on CNF-containing food. Larvae and adult flies reared on CNF-containing food showed dark masses on their body cavities (arrows). Arrowheads indicate melanotic spots in segments of larvae. (D) The number of crystal cells in larvae reared on CNF-containing food and visualized by heat shock-induced melanization increased upon administration of $100 \mu \mathrm{g} / \mathrm{mL}$ of CNFs. $* P<0.05$. (E) The mRNA levels of defensin were analyzed via real-time quantitative polymerase chain reaction. Expression level of defensin was increased upon $100 \mu \mathrm{g} / \mathrm{mL}$ of $\mathrm{CNF}$ administration. $* P<0.05$.

Abbreviation: $\mathrm{CNF}$, carbon nanofiber.

of $100 \mu \mathrm{g} / \mathrm{mL}$ of CNF-supplemented female flies was $33.96 \pm 0.78$ days $(15.67 \%$ increase compared to control, log-rank test, $\left.\chi^{2}=22.22, P<0.0001\right)$. The increased lifespan of $100 \mu \mathrm{g} / \mathrm{mL}$ of CNF-supplemented flies can be attributed to the hormetic effect of mild stress induced by exposure to a low-dose CNF diet (see "Discussion"). To confirm whether or not these beneficial or adverse effects could be attributed specifically to CNFs, we analyzed the survival rate of flies administered graphene, a flat monolayer form of carbon nanomaterials. At the same dose of CNFs, graphene did not 

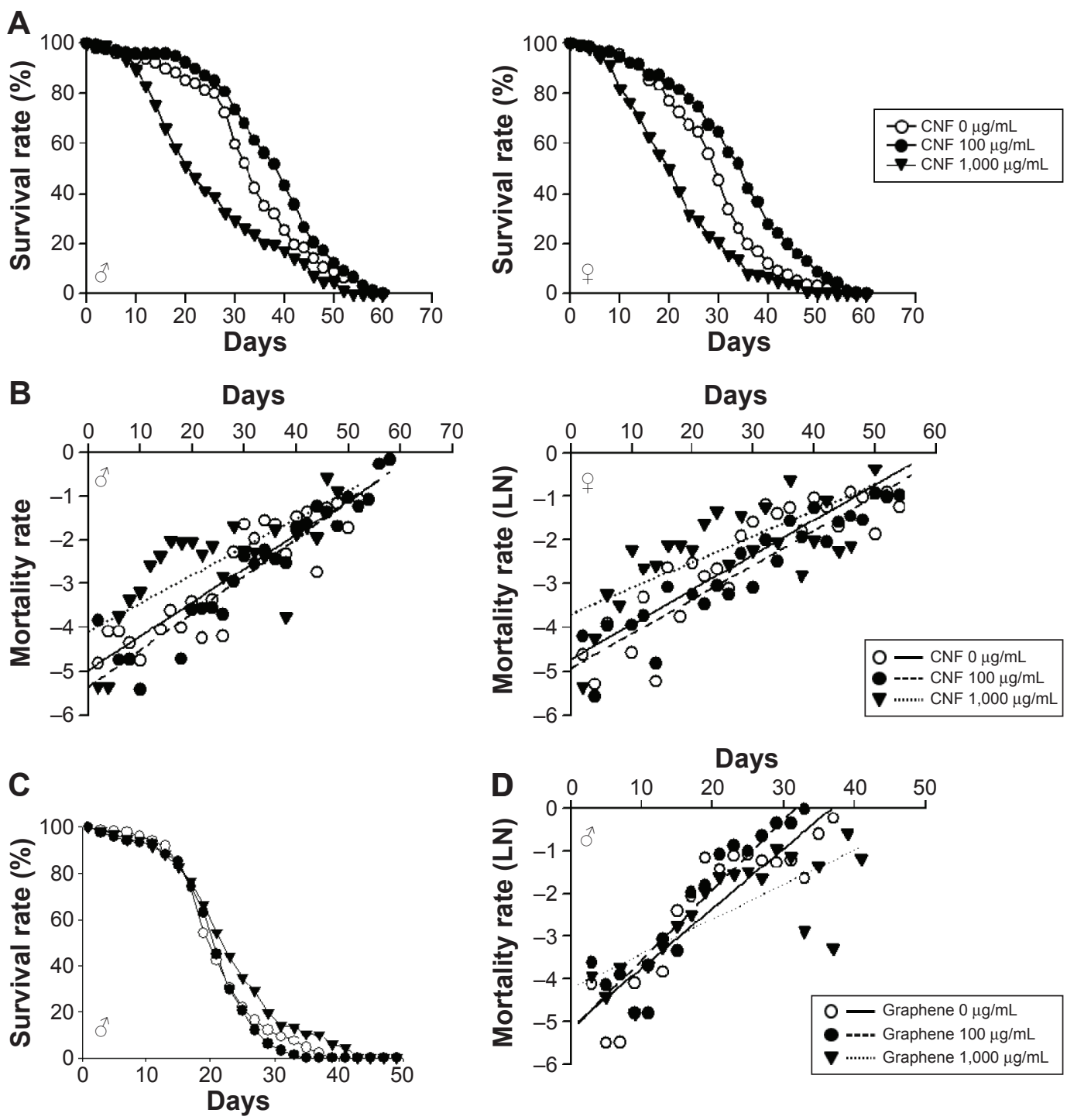

Figure 3 Effects of CNF oral administration on adult viability in Drosophila melanogaster.

Notes: (A) Chronic administration of CNFs at a dose of $1,000 \mu \mathrm{g} / \mathrm{mL}$ reduced survival time of Drosophila (lines with black triangles; male, $P<0.000 \mathrm{I}$; female, $P<0.000 \mathrm{I}$ ). However, the lifespan of flies administered $100 \mu \mathrm{g} / \mathrm{mL}$ of CNFs significantly increased (lines with black circles; male, $P<0.000 \mathrm{I}$; female, $P<0.000 \mathrm{I}$ ). (B) Mortality curves showing age-specific mortality rates of flies administered CNFs. The natural log of the mortality rate was plotted using the Gompertz mortality model. Age-specific mortality rates of both male and female flies administered $1,000 \mu \mathrm{g} / \mathrm{mL}$ of CNFs significantly increased, whereas those of flies administered $100 \mu \mathrm{g} / \mathrm{mL}$ of $\mathrm{CNFs}$ slightly decreased. (C) Administration of graphene at the same dose as CNF did not decrease the survival rate of Drosophila $(\mathrm{male}$ flies, $100 \mu \mathrm{g} / \mathrm{mL}, P<0.1617 ; \mathrm{I}, 000 \mu \mathrm{g} / \mathrm{mL}, 9.52 \%$ increase of mean lifespan, $P<0.000 \mathrm{I})$. (D) Age-specific mortality rate of flies exposed to graphene did not increase at early life stages.

Abbreviation: CNF, carbon nanofiber.

significantly decrease the survival or mortality rate of flies (Figure $3 \mathrm{C}$ and $\mathrm{D}$ ). These results indicate that a high dose of CNF had toxic effects on fly survival. Further, this toxicity was CNF-specific and not a general characteristic of nanomaterials since graphene did not show any toxicity.

\section{Effects of CNFs on Drosophila reproduction, feeding, and locomotion performance}

To further analyze the effects of CNFs on the physiology of Drosophila, we investigated the effects of CNFs on fecundity of flies. The fecundity of flies supplemented with $1,000 \mu \mathrm{g} / \mathrm{mL}$ of CNFs significantly decreased, whereas that of flies supplemented with $100 \mu \mathrm{g} / \mathrm{mL}$ of CNFs was unchanged (Figure $4 \mathrm{~A}$ ). Furthermore, we analyzed food intake by CNF-supplemented flies. The amount of food intake by flies reared on CNFcontaining food significantly decreased in a dose-dependent manner in both sexes. The amount of food ingested by flies reared on $100 \mu \mathrm{g} / \mathrm{mL}$ of CNFs decreased by approximately $30 \%$ compared to the control, whereas that of $1,000 \mu \mathrm{g} / \mathrm{mL}$ of CNF-supplemented flies was only $40 \%-50 \%$ that of the control (Figure 4B). 

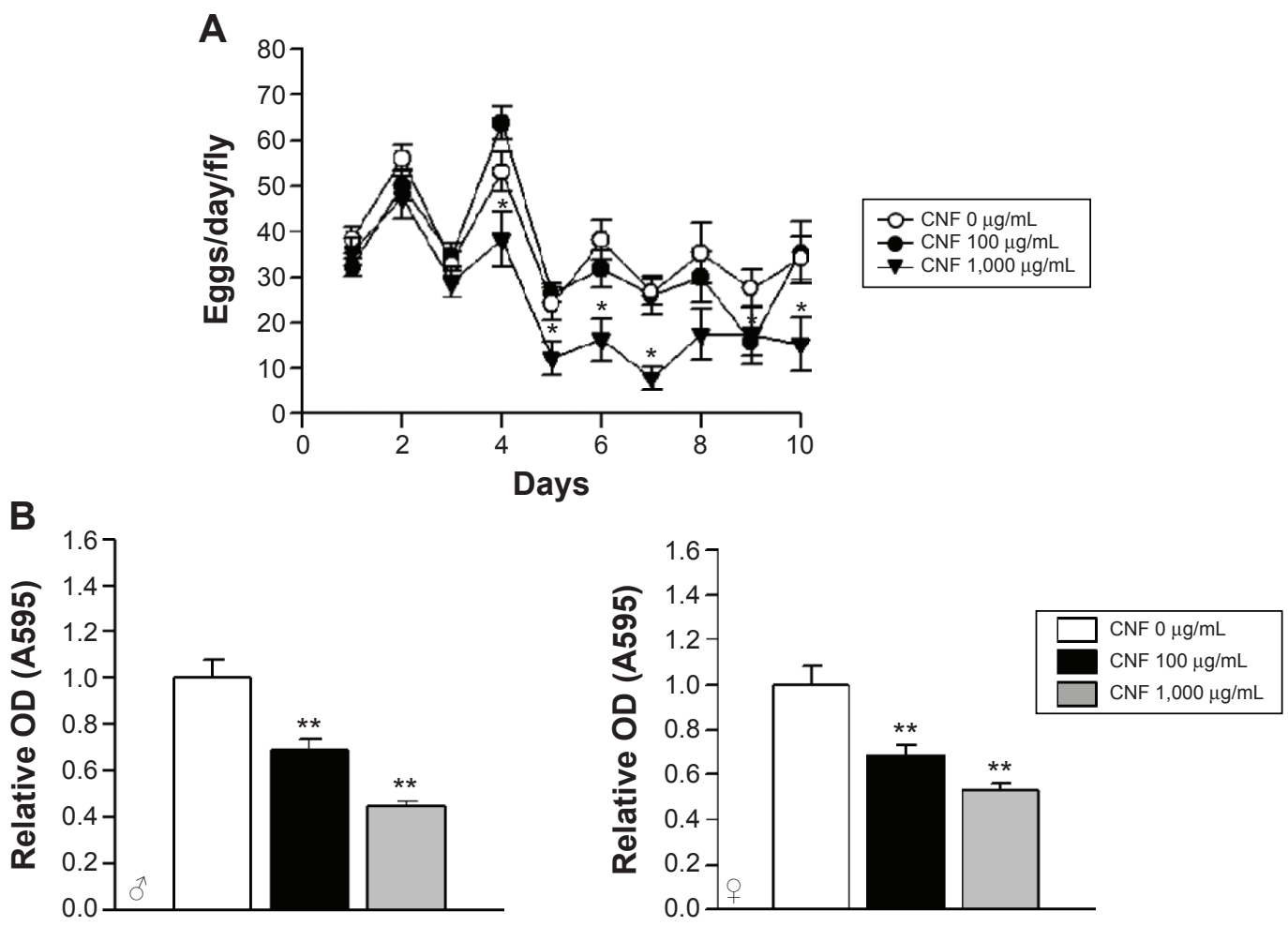

Figure 4 Effects of CNF ingestion on reproduction and feeding in Drosophila melanogaster.

Notes: (A) Reproductive activity of flies was determined based on the number of eggs obtained from a female fly reared on CNF-containing food. Reproductive activity of flies administered I, $000 \mu \mathrm{g} / \mathrm{mL}$ of CNFs significantly decreased from 4 days after eclosion (line with black triangles). Administration of I00 $\mu \mathrm{g} / \mathrm{mL}$ of CNFs did not affect reproductive activity of flies (line with black circles). (B) Food intake by flies was assessed by measuring the amount of blue dye-containing food after I 2 hours. Food intake by flies administered CNFs significantly decreased in a dose-dependent manner. $* P<0.05 ; * * P<0.001$.

Abbreviations: CNF, carbon nanofiber; OD, optical density.

The physical activity of CNF-supplemented flies was also determined by measurement of vertical climbing activity. Supplementation with CNFs did not have any significant effects on physical activity of female flies at both 1 and 3 weeks after eclosion (Figure 5). In male flies supplemented with $1,000 \mu \mathrm{g} / \mathrm{mL}$ of $\mathrm{CNFs}$, climbing activity significantly decreased at 1 week after eclosion, whereas climbing activity upon $100 \mu \mathrm{g} / \mathrm{mL}$ of CNF supplementation actually increased at 3 weeks after eclosion (Figure 5). The activity data for males were shown to be consistent with the lifespan data showing an adverse effect of $1,000 \mathrm{mg} / \mathrm{mL}$ of CNFs and beneficial effect of $100 \mu \mathrm{g} / \mathrm{mL}$ of CNFs.

\section{Effects of CNFs on resistance to starvation stress}

To investigate the effects of CNFs on susceptibility to stress, we assessed the survival rates of flies reared on CNF-containing food under starvation stress conditions. Male flies reared on standard medium survived for $33.2 \pm 0.55$ hours under starvation conditions, whereas male flies reared on $1,000 \mu \mathrm{g} / \mathrm{mL}$ of CNFs survived for $25.6 \pm 0.51$ hours under starvation conditions (Figure 6A) (22.9\% decrease compared to control, log-rank test, $\left.\chi^{2}=82.51, P<0.0001\right)$. In female flies, the mean lifespan of control flies was 51.52 \pm 0.89 hours, whereas that of flies reared on $1,000 \mu \mathrm{g} / \mathrm{mL}$ of CNFs was $28.08 \pm 0.7$ hours under starvation conditions (Figure 6A) (45.5\% decrease compared to control, log-rank test, $\left.\chi^{2}=249.03, P<0.0001\right)$. Supplementation with $100 \mu \mathrm{g} / \mathrm{mL}$ of CNFs did not affect lifespan under starvation stress conditions in male flies (Figure 6A) (log-rank test, $\left.\chi^{2}=2.7519, P=0.097\right)$, whereas the lifespan of female flies slightly decreased (Figure 6A) (11.11\% decrease compared to control, log-rank test, $\left.\chi^{2}=12.33, P<0.001\right)$.

Resistance to starvation mainly depends on fat content, which is correlated with body weight. The reduced survival under starvation conditions may be attributed to the lower body weight of flies reared on CNFs induced by reduction of feeding rate (Figure 4B). Therefore, the body weight of flies reared on CNFs was measured. Interestingly, body mass of newly eclosed male and female flies developed on CNFs was significantly higher than that of the control, and weight of male flies fed CNFs for an additional 7 days was still heavier than that of the control (Figure 6B, C). These results suggest that the reduced survival of flies fed CNFs under starvation conditions was not due to reduced food intake. 
A

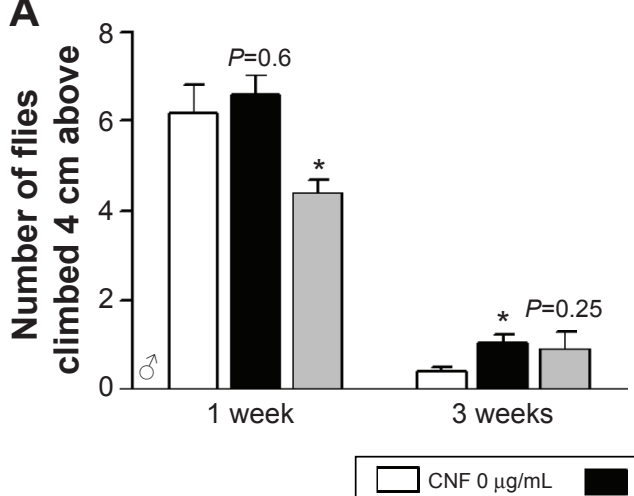

B

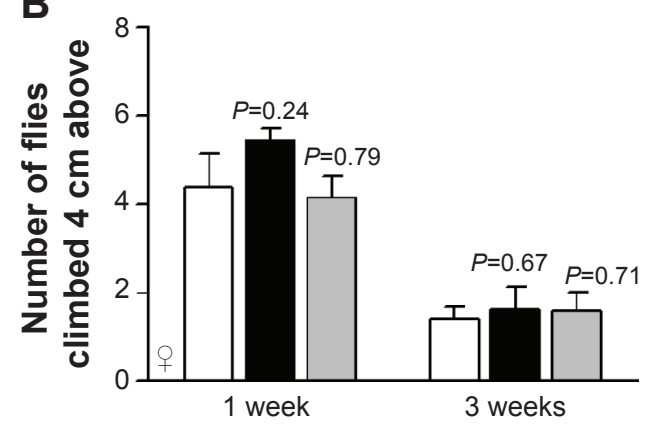

Figure 5 Effects of CNF ingestion on physical activity of Drosophila.

Notes: The physical activity of flies was determined by vertical climbing assay. The physical activity of female flies (A) was not significantly affected by CNF administration. However, the physical activity of male flies (B) significantly decreased at I week after eclosion upon I,000 $\mu \mathrm{g} / \mathrm{mL}$ of $\mathrm{CNF}$ ingestion. Male flies administered I00 $\mu \mathrm{g} / \mathrm{mL}$ of CNFs showed enhanced physical activity at 3 weeks after eclosion. $* P<0.05$.

Abbreviation: CNF, carbon nanofiber.

\section{Induction of ROS by CNF}

\section{supplementation}

The toxicity of nanomaterials has been reported to be mainly associated with generation of ROS. In addition, the observed physiological phenomena caused by exposure to CNFs in this study, such as changes in lifespan and development, are known to be related to internal ROS levels. To analyze the internal ROS level in flies reared on CNF-containing diet, total GSH and GSSG levels were measured using 5,5'dithio-bis-2-nitrobenzoic acid and GSH reductase. ${ }^{37}$ Total amounts of GSH and GSSG in whole flies supplemented with CNFs were calculated from a GSSG standard curve. Flies treated with $20 \mathrm{mM}$ paraquat for 12 hours were used as a positive control. The GSH/GSSG ratio of control flies
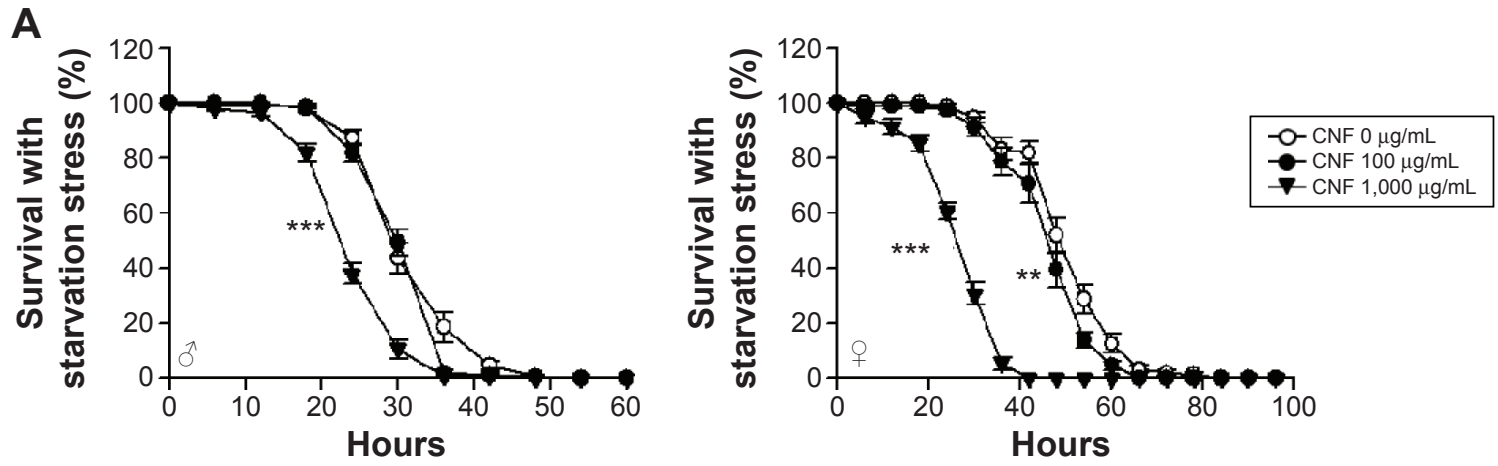

B

C
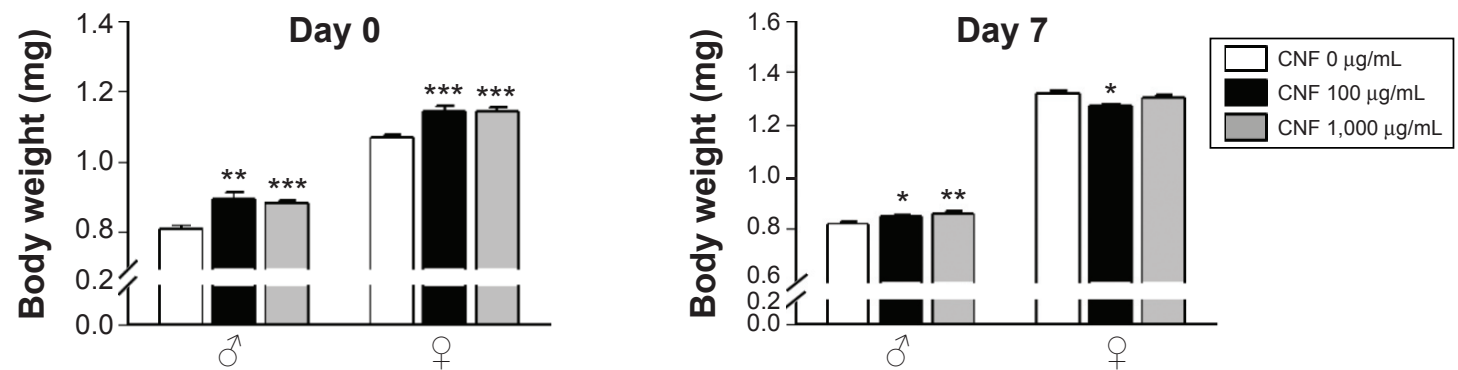

Figure 6 Effects of CNF ingestion on resistance to starvation stress and body weight.

Notes: (A) Survival rate of flies administered $1,000 \mu \mathrm{g} / \mathrm{mL}$ of CNFs significantly decreased under starvation conditions (lines with black triangles, $P<0.000 \mathrm{I}$ ), whereas $100 \mu \mathrm{g} / \mathrm{mL}$ of CNFs had a minor effect or none (lines with black circles). (B) Body weight of newly hatched flies is shown. Newly eclosed flies reared on CNF-containing food were heavier than the control. (C) Body weight of 7 -day-old flies is shown. Body weight of male flies administered CNFs for 7 days still significantly increased. $* P<0.05$; $* * P<0.001 ; * * * P<0.0001$.

Abbreviation: CNF, carbon nanofiber. 
was 3.65 \pm 0.53 , whereas ratios of flies supplemented with 100 and $1,000 \mu \mathrm{g} / \mathrm{mL}$ of CNFs were $2.83 \pm 0.19$ and $1.75 \pm 0.26$, respectively (Figure 7A). The reduced GSH/GSSG ratio indicates that oral administration of CNFs generated oxidative stress in Drosophila.

Under oxidative stress conditions, the antioxidant and detoxification systems of organisms are activated for protection. To investigate the effects of CNF administration on expression of antioxidant enzymes, mRNA levels of $S O D$ and $C a t$ were analyzed using real-time qPCR. Interestingly, transcription levels of cytosolic CuZnSOD (SOD1) increased in whole flies administered $100 \mu \mathrm{g} / \mathrm{mL}$ of CNFs, whereas expression levels of mitochondrial MnSOD (SOD2) and Cat decreased upon $1,000 \mu \mathrm{g} / \mathrm{mL}$ of CNF supplementation (Figure 7B). These results suggest that a high dose of CNFs $(1,000 \mu \mathrm{g} / \mathrm{mL})$ disturbed the antioxidant system of flies, whereas a low dose of CNFs $(100 \mu \mathrm{g} / \mathrm{mL})$ actually stimulated the antioxidant system along with mild generation of ROS.

\section{Discussion}

Increased attention toward use of nanomaterials by engineers and biomedical scientists has raised concerns over their toxicity in human health and the environment. In the current study, we analyzed the toxicity of chronic exposure to CNFs using D. melanogaster as an in vivo model system.

Long-term oral administration of CNFs had dose-dependent distinct effects on Drosophila physiology in this study. CNFs showed significant adverse effects on Drosophila at a concentration of $1,000 \mu \mathrm{g} / \mathrm{mL}$, including reduced larval viability based on the pupa:egg ratio, survival of adult flies, reproductive activity, climbing ability, and survival under starvation stress conditions. Several reports have showed that oral administration of nanomaterials has adverse effects on Drosophila physiology. Especially, silver and gold nanoparticles show toxic effects on Drosophila fecundity and lifespan. ${ }^{21-23,29}$ On the other hand, other studies reported that nanomaterials lack any significant adverse effects on Drosophila. For example, multi-walled carbon nanotubes at a concentration of $1,000 \mu \mathrm{g} / \mathrm{g}$ were shown to have no significant effect on developmental time or egg viability, ${ }^{31}$ and single-walled carbon nanotubes at a concentration of 12-24 ppm during larval stage were reported to have no effect on pupal viability. ${ }^{30}$ Further, carbon nanotubes functionalized with a hydroxyl group were reported to not affect the eclosion rate of flies. ${ }^{24}$ Assuming a food intake of $1.5 \mu \mathrm{L}$ per day, ${ }^{38}$ we speculate that the maximum amount of CNFs consumed by individual flies was 0.15 or $1.5 \mu \mathrm{g} / \mathrm{fly} /$ day in this study, not considering reduction of feeding rate (Figure 3B). Although the amount of CNFs in our fly diet was higher than naturally occurring levels, it is plausible for humans to ingest such abundant amounts of nanomaterials since continuous ingestion of contaminated foods or water is the most common route of nanomaterial ingestion. ${ }^{39}$ Therefore, our data showing the obvious toxic effects of CNFs on Drosophila physiology provide valuable information for the study of human health.

The adverse effects of chronic exposure to a high dose of CNFs $(1,000 \mu \mathrm{g} / \mathrm{mL})$ are assumed to be the result of direct toxicity. However, we cannot rule out the possibility of indirect toxicological effects caused by reduced food intake. Chronic oral ingestion of CNFs reduced food intake in flies

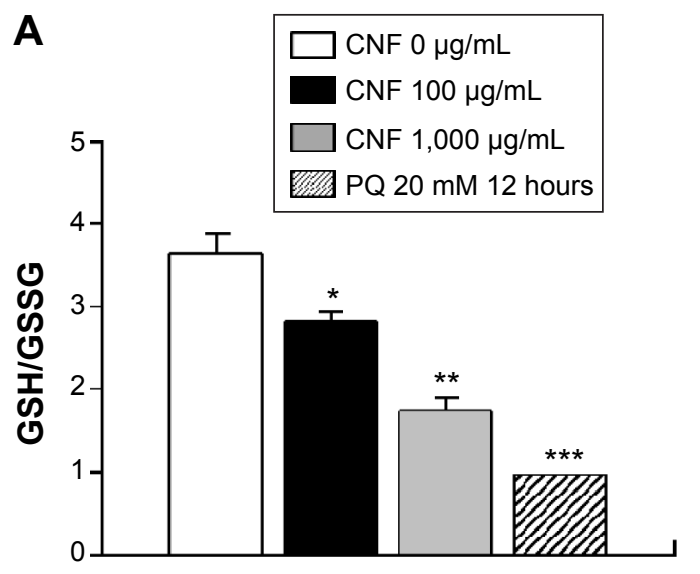

B

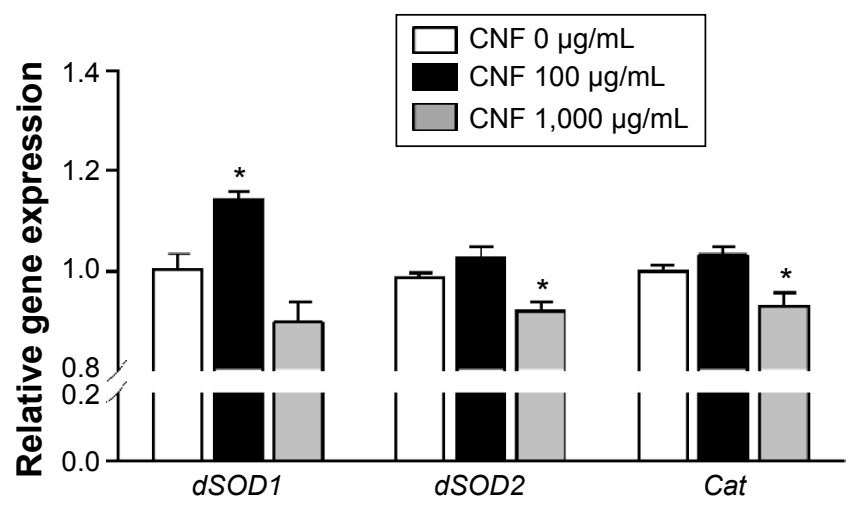

Figure 7 CNF-induced oxidative stress in Drosophila.

Notes: (A) The intracellular reactive oxygen species level was analyzed by measuring the ratio of GSH to GSSG. The GSH/GSSG ratio significantly decreased upon chronic administration of CNFs to Drosophila. Treatment with $20 \mathrm{mM}$ PQ was used as a positive control. (B) The mRNA levels of antioxidant enzymes were analyzed via real-time quantitative polymerase chain reaction. Expression levels of $d S O D I$, dSOD2, and catalase increased upon $100 \mu \mathrm{g} / \mathrm{mL}$ of $\mathrm{CNF}$ administration but decreased upon I,000 $\mu \mathrm{g} / \mathrm{mL}$ of CNF administration. $* P<0.05 ; * * P<0.001 ; * * * P<0.000$ I.

Abbreviations: CNF, carbon nanofiber; GSH, glutathione; GSSG, oxidized glutathione; PQ, paraquat. 
by up to $60 \%$. Reduction of food intake can inhibit reproduction and decrease body weight, subsequently impairing physical activity. ${ }^{40,41}$ In addition, reduction of stored nutrients increases sensitivity to starvation stress, which ultimately reduces the survival rate. ${ }^{42}$ Body weight data suggest that reduced food intake did not increase sensitivity to starvation resistance, as body weight did not decrease in CNF-fed flies showing reduced survival under starvation resistance (Figure 5). Interestingly, newly eclosed male and female flies developed on CNF-containing food were significantly heavier compared to the control. This is possibly due to the delayed development of larvae (data not shown), increasing the feeding period during larval development.

Surprisingly, a low dose of CNFs $(100 \mu \mathrm{g} / \mathrm{mL})$ showed beneficial effects on the physiology of flies. Ingestion of CNFs $(100 \mu \mathrm{g} / \mathrm{mL})$ did not have any significant effect on developmental rate or fecundity. Interestingly, lifespan, climbing activity, and resistance to oxidative stress (data not shown) were improved by long-term oral administration of $100 \mu \mathrm{g} / \mathrm{mL}$ of CNFs. The beneficial effects of $100 \mu \mathrm{g} / \mathrm{mL}$ of CNF supplementation can be explained by reduced food intake. Mild reduction of nutrient intake without malnutrition, known as dietary/calorie restriction, is known to have beneficial effects such as prolonged lifespan and increased resistance to several stresses. ${ }^{43}$ In addition, independent of reduced food intake, our results can be explained by the hormetic effect, which is a biphasic dose response with a low-dose beneficial effect and high-dose toxic effects. ${ }^{44}$ Since dietary/calorie restriction is also considered as a mild stress and its beneficial effects are due to the hormetic effect, ${ }^{45}$ it can be assumed that long-term exposure to a low dose of CNFs functions as a mild stress to activate the defense system in flies against subsequent stresses.

Generation of ROS and subsequent induction of oxidative stress upon exposure to nanomaterials is considered to be the most important toxicity mechanism of nanomaterials. ${ }^{13}$ The ROS level measured based on the GSH/GSSG ratio increased in flies fed CNFs in a dose-dependent manner, and expression of some antioxidant enzyme(s) increased with a low dose of CNFs $(100 \mu \mathrm{g} / \mathrm{mL})$ but decreased with a high dose of CNFs $(1,000 \mu \mathrm{g} / \mathrm{mL})$. Mild oxidative stress stimulates the defense system, including antioxidant and detoxification enzymes, but this protective response is perturbed and overtaken by inflammation and cytotoxicity at higher levels of oxidative stress. ${ }^{46,47}$ Thus, a low dose of CNFs may function as a mild stress in flies to activate the defense response, whereas a high dose of CNFs may perturb the defense response, reducing the expression of some antioxidant enzymes.
In summary, long-term oral administration of a high dose of CNFs has obvious toxic effects on fly physiology, possibly due to impairment of feeding performance and antioxidant systems along with enhanced intracellular ROS production. In addition, exposure to a low dose of CNFs has beneficial effects on fly physiology, especially resistance to oxidative stress mediated by enhanced antioxidant defense.

\section{Acknowledgments}

This research was supported by the Basic Science Research Program through the National Research Foundation of Korea (KRF), funded by the Ministry of Education (number 2013R1A1A2063664).

\section{Disclosure}

The authors report no conflicts of interest in this work.

\section{References}

1. Harrison BS, Atala A. Carbon nanotube applications for tissue engineering. Biomaterials. 2007;28(2):344-353.

2. De Volder MF, Tawfick SH, Baughman RH, Hart AJ. Carbon nanotubes: present and future commercial applications. Science. 2013;339(6119): 535-539.

3. Mundra RV, Wu X, Sauer J, Dordick JS, Kane RS. Nanotubes in biological applications. Curr Opin Biotechnol. 2014;28:25-32.

4. Rastogi V, Yadav P, Bhattacharya SS, et al. Carbon nanotubes: an emerging drug carrier for targeting cancer cells. J Drug Deliv. 2014; 2014:670815.

5. Jang S, Jang H, Lee Y, et al. Flexible, transparent single-walled carbon nanotube transistors with graphene electrodes. Nanotechnology. 2010; 21(42):425201.

6. Donaldson K, Poland CA, Murphy FA, MacFarlane M, Chernova T, Schinwald A. Pulmonary toxicity of carbon nanotubes and asbestos similarities and differences. Adv Drug Deliv Rev. 2013;65(15):2078-2086.

7. Castranova V, Schulte PA, Zumwalde RD. Occupational nanosafety considerations for carbon nanotubes and carbon nanofibers. Acc Chem Res. 2013;46(3):642-649.

8. Jong KPD, Geus JW. Carbon nanofibers: catalytic synthesis and applications. Catal Rev. 2000;42(4):481-510.

9. Darne C, Terzetti F, Coulais C, et al. In vitro cytotoxicity and transforming potential of industrial carbon dust (fibers and particles) in syrian hamster embryo (SHE) cells. Ann Occup Hyg. 2010;54(5):532-544.

10. Jain S, Webster TJ, Sharma A, Basu B. Intracellular reactive oxidative stress, cell proliferation and apoptosis of Schwann cells on carbon nanofibrous substrates. Biomaterials. 2013;34(21):4891-4901.

11. Kisin ER, Murray AR, Sargent L, et al. Genotoxicity of carbon nanofibers: are they potentially more or less dangerous than carbon nanotubes or asbestos? Toxicol Appl Pharmacol. 2011;252(1):1-10.

12. Green M, Howman E. Semiconductor quantum dots and free radical induced DNA nicking. Chem Commun (Camb). 2005(1):121-123.

13. Nel A, Xia T, Mädler L, Li N. Toxic potential of materials at the nanolevel. Science. 2006;311(5761):622-627.

14. Monteiller C, Tran L, MacNee W, et al. The pro-inflammatory effects of low-toxicity low-solubility particles, nanoparticles and fine particles, on epithelial cells in vitro: the role of surface area. Occup Environ Med. 2007;64(9):609-615.

15. Ahamed M, Posgai R, Gorey TJ, Nielsen M, Hussain SM, Rowe JJ. Silver nanoparticles induced heat shock protein 70 , oxidative stress and apoptosis in Drosophila melanogaster. Toxicol Appl Pharmacol. 2010; 242(3):263-269. 
16. Posgai R, Cipolla-McCulloch CB, Murphy KR, Hussain SM, Rowe JJ, Nielsen MG. Differential toxicity of silver and titanium dioxide nanoparticles on Drosophila melanogaster development, reproductive effort, and viability: size, coatings and antioxidants matter. Chemosphere. 2011;85(1):34-42.

17. Ramkumar KM, Manjula C, Gnanakumar G, et al. Oxidative stressmediated cytotoxicity and apoptosis induction by $\mathrm{TiO}_{2}$ nanofibers in HeLa cells. Eur J Pharm Biopharm. 2012;81(2):324-333.

18. Ong C, Yung LY, Cai Y, Bay BH, Baeg GH. Drosophila melanogaster as a model organism to study nanotoxicity. Nanotoxicology. Epub 2014 Jul 22.

19. Lee HY, Lee SH, Min KJ. Insects as a model system for aging studies. Entomological Research. 2015.

20. Demir E, Vales G, Kaya B, Creus A, Marcos R. Genotoxic analysis of silver nanoparticles in Drosophila. Nanotoxicology. 2011;5(3): $417-424$.

21. Armstrong N, Ramamoorthy M, Lyon D, Jones K, Duttaroy A. Mechanism of silver nanoparticles action on insect pigmentation reveals intervention of copper homeostasis. PLoS One. 2013;8(1):e53186.

22. Pompa PP, Vecchio G, Galeone A, et al. In vivo toxicity assessment of gold nanoparticles in Drosophila melanogaster. Nano Res. 2011;4(4): 405-413.

23. Vecchio G, Galeone A, Brunetti V, et al. Concentration-dependent, size-independent toxicity of citrate capped AuNPs in Drosophila melanogaster. PLoS One. 2012;7(1):e29980.

24. Philbrook NA, Walker VK, Afrooz AR, Saleh NB, Winn LM. Investigating the effects of functionalized carbon nanotubes on reproduction and development in Drosophila melanogaster and CD-1 mice. Reprod Toxicol. 2011;32(4):442-448.

25. Adolfsson K, Schneider M, Hammarin G, Häcker U, Prinz CN. Ingestion of gallium phosphide nanowires has no adverse effect on Drosophila tissue function. Nanotechnology. 2013;24(28):285101.

26. Barandeh F, Nguyen PL, Kumar R, et al. Organically modified silica nanoparticles are biocompatible and can be targeted to neurons in vivo. PLoS One. 2012;7(1):e29424.

27. Goyal R, Tripathi SK, Tyagi S, et al. Gellan gum-PEI nanocomposites as efficient gene delivery agents. J Biomed Nanotechnol. 2011;7(1): 38-39.

28. Fangueiro JF, Gonzalez-Mira E, Martins-Lopes P, et al. A novel lipid nanocarrier for insulin delivery: production, characterization and toxicity testing. Pharm Dev Technol. 2013;18(3):545-549.

29. Panacek A, Prucek R, Safarova D, et al. Acute and chronic toxicity effects of silver nanoparticles (NPs) on Drosophila melanogaster. Environ Sci Technol. 2011;45(11):4974-4979.

30. Leeuw TK, Reith RM, Simonette RA, et al. Single-walled carbon nanotubes in the intact organism: near-IR imaging and biocompatibility studies in Drosophila. Nano Lett. 2007;7(9):2650-2654.
31. Liu X, Vinson D, Abt D, Hurt RH, Rand DM. Differential toxicity of carbon nanomaterials in Drosophila: larval dietary uptake is benign, but adult exposure causes locomotor impairment and mortality. Environ Sci Technol. 2009;43(16):6357-6363.

32. Mueller LD, Nusbaum TJ, Rose MR. The Gompertz equation as a predictive tool in demography. Exp Gerontol. 1995;30(6):553-569.

33. Seong KM, Kim CS, Seo SW, et al. Genome-wide analysis of low-dose irradiated male Drosophila melanogaster with extended longevity. Biogerontology. 2011;12(2):93-107.

34. Tsuda M, Ootaka R, Ohkura C, et al. Loss of Trx-2 enhances oxidative stress-dependent phenotypes in Drosophila. FEBS Lett. 2010;584(15): 3398-3401.

35. Shen LR, Xiao F, Yuan P, et al. Curcumin-supplemented diets increase superoxide dismutase activity and mean lifespan in Drosophila. Age (Dordr). 2013;35(4):1133-1142.

36. Biteau B, Hochmuth CE, Jasper H. JNK activity in somatic stem cells causes loss of tissue homeostasis in the aging Drosophila gut. Cell Stem Cell. 2008;3(4):442-455.

37. Pal AK, Hsieh SF, Khatri M, et al. Screening for oxidative damage by engineered nanomaterials: a comparative evaluation of FRAS and DCFH. J Nanopart Res. 2014;16:2167.

38. Deshpande SA, Carvalho GB, Amador A, et al. Quantifying Drosophila food intake: comparative analysis of current methodology. Nat Methods. 2014;11(5):535-540.

39. Wang H, Wang J, Deng X, et al. Biodistribution of carbon singlewall carbon nanotubes in mice. J Nanosci Nanotechnol. 2004;4(8): 1019-1024.

40. Bross TG, Rogina B, Helfand SL. Behavioral, physical, and demographic changes in Drosophila populations through dietary restriction. Aging Cell. 2005;4(6):309-317.

41. O'Brien DM, Min KJ, Larsen T, Tatar M. Use of stable isotopes to examine how dietary restriction extends Drosophila lifespan. Curr Biol. 2008;18(4):R155-R156.

42. Min KJ, Tatar M. Restriction of amino acids extends lifespan in Drosophila melanogaster. Mech Ageing Dev. 2006;127(7):643-646.

43. Lee SH, Min KJ. Caloric restriction and its mimetics. BMB Rep. 2013; 46(4):181-187.

44. Kirkwood TB, Shanley DP. Caloric restriction, hormesis and life history plasticity. Hum Exp Toxicol. 2000;19(6):338-339.

45. Martins I, Galluzzi L, Kroemer G. Hormesis, cell death and aging. Aging (Albany NY). 2011;3(9):821-828.

46. Chakraborty PK, Mustafi SB, Raha S. Pro-survival effects of repetitive low-grade oxidative stress are inhibited by simultaneous exposure to Resveratrol. Pharmacol Res. 2008;58(5-6):281-289.

47. Sen P, Mukherjee S, Bhaumik G, et al. Enhancement of catalase activity by repetitive low-grade $\mathrm{H} 2 \mathrm{O} 2$ exposures protects fibroblasts from subsequent stress-induced apoptosis. Mutat Res. 2003;529(1-2):87-94.
International Journal of Nanomedicine

\section{Publish your work in this journal}

The International Journal of Nanomedicine is an international, peerreviewed journal focusing on the application of nanotechnology in diagnostics, therapeutics, and drug delivery systems throughout the biomedical field. This journal is indexed on PubMed Central, MedLine, CAS, SciSearch $®$, Current Contents $\AA /$ Clinical Medicine,

\section{Dovepress}

Journal Citation Reports/Science Edition, EMBase, Scopus and the Elsevier Bibliographic databases. The manuscript management system is completely online and includes a very quick and fair peer-review system, which is all easy to use. Visit http://www.dovepress.com/ testimonials.php to read real quotes from published authors. 